\title{
A Framework for Application-Guided Task Management on Heterogeneous Embedded Systems
}

\author{
FRANCISCO GASPAR, LUIS TANIÇA, PEDRO TOMÁS, ALEKSANDAR ILIC, \\ and LEONEL SOUSA, INESC-ID, Instituto Superior Técnico, Universidade de Lisboa
}

\begin{abstract}
In this article, we propose a general framework for fine-grain application-aware task management in heterogeneous embedded platforms, which allows integration of different mechanisms for an efficient resource utilization, frequency scaling, and task migration. The proposed framework incorporates several components for accurate runtime monitoring by relying on the OS facilities and performance self-reporting for parallel and iterative applications. The framework efficiency is experimentally evaluated on a real hardware platform, where significant power and energy savings are attained for SPEC CPU2006 and PARSEC benchmarks, by guiding frequency scaling and intercluster migrations according to the runtime application behavior and predefined performance targets.
\end{abstract}

CCS Concepts: $\bullet$ Computer systems organization $\rightarrow$ Multicore architectures; Heterogeneous (hybrid) systems; $\bullet$ Software and its engineering $\rightarrow$ Process management;

Additional Key Words and Phrases: Heterogeneous multiprocessor, scheduling, embedded systems, quality of service, big.LITTLE, task migration, dynamic voltage and frequency control

ACM Reference Format:

Francisco Gaspar, Luis Taniça, Pedro Tomás, Aleksandar Ilic, and Leonel Sousa. 2015. A framework for application-guided task management on heterogeneous embedded systems. ACM Trans. Archit. Code Optim. 12, 4, Article 42 (December 2015), 25 pages.

DOI: http://dx.doi.org/10.1145/2835177

\section{INTRODUCTION}

Multicore processors are currently moving towards heterogeneou designs to improve overall chip energy efficiency, such as embedded common ISA architectures with several multicore clusters. By strictly relying on hardware solutions, the efficiency of these multicore architectures can hardly be maintained in the presence of current technological constraints [Esmaeilzadeh et al. 2011]. A much tighter interrelation between the software and hardware layers is essential to fully exploit the capabilities of heterogeneous platforms. However, efficient software solutions are yet to be devised, especially at the Operating System (OS) level [Pitre 2015].

Modern Linux-based OSs rely on two decoupled subsystems to manage task execution, that is, the task scheduler based on the default Completely Fair Scheduler (CFS) class and several Dynamic Voltage and Frequency Scaling (DVFS) subsystems. In particular, the task scheduler defines how the running tasks are assigned to the computational resources, while DVFS aims at providing power/energy savings by adjusting

The work presented herein was partially supported by national funds through Fundação para a Ciência e a Tecnologia (FCT) under project P2HCS (ref. PTDC/EEI-ELC/3152/2012) and project UID/CEC/50021/2013. Authors' addresses: Rua Alves Redol 9, 1000-029 Lisbon, Portugal; email: \{Francisco.Gaspar, Luis.Tanica, Pedro.Tomas, Aleksandar.Ilic, Leonel.Sousa\}@inesc-id.pt.

Permission to make digital or hard copies of part or all of this work for personal or classroom use is granted without fee provided that copies are not made or distributed for profit or commercial advantage and that copies show this notice on the first page or initial screen of a display along with the full citation. Copyrights for components of this work owned by others than ACM must be honored. Abstracting with credit is permitted. To copy otherwise, to republish, to post on servers, to redistribute to lists, or to use any component of this work in other works requires prior specific permission and/or a fee. Permissions may be requested from Publications Dept., ACM, Inc., 2 Penn Plaza, Suite 701, New York, NY 10121-0701 USA, fax +1 (212) 869-0481, or permissions@acm.org.

(c) 2015 ACM 1544-3566/2015/12-ART42 $\$ 15.00$

DOI: http://dx.doi.org/10.1145/2835177 
the operating voltage and frequency. To mitigate the limitations of the default CFS when applied to heterogenous platforms (e.g., ARM big.LITTLE) [Pitre 2014], several industry-standard approaches are proposed, such as In-Kernel Switcher (IKS) [Poirier 2013] and Global Task Scheduling (GTS) [Jeff 2013]. However, these approaches mainly target intercluster task migration by only slightly amending the original CFS functionality, while the DVFS decisions are still derived without almost any coordination with the scheduler. Thus, they might not be capable of ensuring the most adequate scheduling decisions for all execution scenarios [Muckle 2014; Pitre 2014]. Currently, CFS+IKS represents the only officially accepted (mainstream) approach, while efficient solutions are yet to be derived [Rasmussen 2014].

To attain efficient execution in heterogeneous systems, current state-of-the-art approaches advocate extending the CFS+DVFS functionality to explicitly consider the application runtime behavior and requirements, for example, via stall-based characterization [Spiliopoulos et al. 2011; Van Craeynest et al. 2012] or the Heartbeats API [Hoffmann et al. 2010]. In heterogeneous embedded systems, only rare attempts have been made to holistically address energy-efficient task management for single-threaded [Muthukaruppan et al. 2013, 2014] and parallel applications [Gaspar et al. 2014; Imes et al. 2015]. However, the ability of these approaches to attain fine-grain thread-level execution control is restricted by the Heartbeats API limited monitoring capabilities [Hoffmann et al. 2010; Sironi et al. 2012], since it does not provide detailed and accurate OS-level profiling information.

For these reasons, a novel general framework for fine-grain application-aware task management in heterogeneous embedded systems is proposed herein, which incorporates several key contributions:

-Performance self-reporting mechanism for parallel and iterative applications through a specifically developed API

- Highly accurate runtime monitoring that relies on OS scheduling facilities to provide detailed performance information on a per-application and a per-task basis

-Unification of decoupled scheduling subsystems based on the information provided by the framework, such as share allocation, frequency scaling, and task migration

The proposed framework also allows amending the original CFS/IKS+DVFS decisions according to the performance requirements of multithreaded applications, which is an approach considered herein to fully demonstrate its functionality. For this purpose, a set of application-aware task management mechanisms are specifically developed and integrated in the proposed framework. For soft real-time iterative applications with specified performance targets, these mechanisms allow achieving power/energy savings in heterogeneous embedded systems by controlling the share allocations, frequency scaling, resource utilization, and intra- and intercluster task migrations.

The remainder of this article is organized as follows: Section 2 provides an extensive overview of the state-of-the-art approaches. Section 3 details the functionality of the proposed framework, while the proposed application-aware task management mechanisms are introduced in Section 4. In Section 5, the efficiency of the proposed framework and system managers is experimentally evaluated on a real hardware platform for a set of single- and multithreaded applications from PARSEC and SPEC CPU 2006 suites. Section 6 concludes the article by summarizing its main contributions.

\section{RELATED WORK}

In modern Linux-based OSs, the functionality of the default task scheduler and DVFS is mainly decoupled and driven by utilization. As a result, these mechanisms get little information at the application level, which may significantly limit their ability to efficiently manage the execution according to the quality-of-service requirements of the 
running applications. In particular, these facilities are unaware of both the application performance targets and the attained performance levels. Accordingly, they usually lead to race-to-idle strategies, which, for many embedded and mobile computing platforms, are not energy efficient [Imes and Hoffmann 2015].

\subsection{Literature Overview}

Several task scheduling techniques have been proposed, both targeting homogeneous and heterogeneous systems and ranging from processor demand to Quality of Service (QoS) approaches, which rely on the development of either new kernel schedulers or user-level frameworks. Although the complete set is extensive, the following paragraphs focus on the most relevant contributions from multiple research fields.

In particular, Brandenburg [2011] and Calandrino et al. [2006] developed LITMUS, a real-time extension of the Linux kernel, and also made important contributions by evaluating a wide range of scheduling plugins. As a consequence, the authors conclude that the Linux scheduling methodology may lead to unnecessary task migrations between cores, significantly increasing computational overheads under strong cache contention, therefore wasting power. However, although these works consider neither heterogeneous processing platforms nor applications with phase changes and/or with variable workloads, it is concluded that global task scheduling algorithms are best under soft real-time constraints.

To tackle application phase changes, Sawalha et al. [2011] proposed a method that relies on online phase characterization in order to predict the best task-to-core mapping whenever the phase resurges again along the execution. Another technique was proposed by Nie and Duan [2012], which relies on the number of stalls per instruction to express the thread efficiency (when running on fast cores) and to guide the thread allocations to a set of heterogeneous cores. However, it does not take into account microarchitectural differences or the task performance information. Van Craeynest et al. [2012] proposed a way to estimate the performance impact of migrating applications across heterogeneous cores. Although lightweight, this approach requires specific hardware support and therefore cannot be applied in general. Hence, the approaches from Nie and Duan [2012] and Van Craeynest et al. [2012] were only validated through cycle-accurate simulators (thus disregarding the influence of OS schedulers) and they require access to performance counters (which are not available on many embedded systems, such as the herein considered Odroid XU+E board).

To attain energy savings, Su et al. [2014] proposed an approach based on stall cycles when fetching the data from out-of-core memory levels. However, this approach relies on a set of performance counters (available only on some AMD processors) to model application performance and energy consumption. A similar approach was proposed in Spiliopoulos et al. [2011] by estimating stall cycles from L2 cache misses. However, neither approach tackles the problem of application scheduling for heterogeneous multicore clusters or considers complex workloads composed of multiple concurrent single and multithreaded applications (for which accurate OS-level monitoring is required).

Several strategies were also proposed to achieve energy savings in an IBM POWER7+ multicore system by managing task-to-core allocations [Vega et al. 2013a] and DVFS [Vega et al. 2013b]. These strategies rely on performance counters to measure the percentage of idle cycles and determine the core utilization, which is used for DVFS and core (un)folding. However, they follow an online trial-and-error approach, which may lead to erroneous decisions that require corrections in later iterations.

The problem of energy budgeting for multicore systems has also been investigated in Cochran et al. [2011] and Ma et al. [2011]. The approach proposed in Cochran et al. [2011] uses a set of offline-built lookup tables to estimate the task-to-core placements and core operating frequency, while the approach of Ma et al. [2011] relies on a 
three-step procedure to control power budgets and per-core frequency levels. However, these approaches do not consider heterogeneous platforms and rely on the existence of performance counters.

Alternative approaches target the problem of guaranteeing application QoS. In particular, Sironi et al. [2012] proposed the integration of a monitoring infrastructure into the Linux kernel and a Performance-Aware Fair Scheduler to adapt scheduling decisions according to the application performance targets. However, no consideration was made regarding workload distribution across heterogeneous cores or about frequency scaling. To tackle this problem, Muthukaruppan et al. [2013] designed an integrated solution that relies on the Application Heartbeats [Hoffmann et al. 2010] and a proportional-integral-derivative controller. This approach adjusts the application time share and core operating frequency according to QoS targets, while also migrating the tasks between ARM A15 and A7 cores to attain energy savings. To improve task distribution efficiency on heterogeneous platforms, a new methodology was proposed based on price theory [Muthukaruppan et al. 2014]. However, this approach requires several iterations to attain stability and it is not evaluated for multithreaded workloads.

Another strategy for soft real-time applications was proposed in Hoffmann et al. [2013] and Imes et al. [2015]. However, by only relying on the Heartbeats API, it does not consider OS-level scheduling information. Furthermore, while Hoffmann et al. [2013] do not tackle system heterogeneity, Imes et al. [2015] require prior information about application performance and power consumption for each possible system configuration. The work from Zhu et al. [2015] relies on event-driven scheduling methodology, but it requires building offline power and performance models, which neglect application phase changes that influence the QoS experience.

\subsection{Modern OS Scheduling for Heterogeneous Systems}

To perform task scheduling, current Linux-based OSs rely on a hierarchy of scheduling classes. While real-time tasks are handled with a fixed-priority policy supporting 100 different priority levels, the CFS class is developed to suit a majority of workloads. However, frequency scaling is decoupled from scheduling decisions and performed via different DVFS subsystems, such as a set of cpufreq governors (see Section 5).

Since the CFS class is originally developed for symmetric (homogenous) systems, it often exhibits significant drawbacks when applied to heterogeneous architectures with several different multicore clusters, such as ARM big.LITTLE [Pitre 2014]. For this purpose, several approaches were proposed, such as In-Kernel Switcher (IKS) [Poirier 2013], Global Task Scheduling (GTS) [Jeff 2013], and Qualcomm hmp scheduling (QuIC) [Muckle 2014]. These approaches mainly target intercluster task migration by only slightly amending the original CFS functionality.

IKS [Poirier 2013] preserves the assumed CFS system symmetry by applying it to a set of virtual cores (each with a "big" and a "little" physical core). Since only one physical core in each pair is allowed to be active (controlled by the virtual clock frequency), only half of the cores can be simultaneously used. To expose all available CPU resources, GTS [Jeff 2013; Poirier 2014] was developed to handle the intercluster migrations, while the original CFS was applied within a cluster. The GTS task-to-cluster allocations are performed by relying on per-entity load tracking (PELT) [Rasmussen 2013] and two predefined threshold values (one for each cluster). In GTS, PELT is used to provide a high-level task classification, where high-intensity workloads are typically assigned to the "big" cores and low-intensity tasks to the "little" ones.

Although power savings can be achieved by packing low-intensity tasks on "little" clusters, the PELT-based techniques were the subject of high criticism in the scheduling community due to a lack of clear evidence on achievable energy savings [Corbet 2013]. Furthermore, the slow responsiveness of PELT geometric-weighted progression 
requires a significant amount of time to discover the correct placement for periodic workloads (of both high and low intensity) [Muckle 2014]. In addition, PELT is a frequency invariant metric, which may severely impact load-balancing decisions due to a distorted perception of the task load at different frequency levels [Rasmussen 2013]. To tackle these drawbacks, the Qualcomm-specific QuIC approach [Muckle 2014] advocates the window-based load tracking, where architecture-specific parameters are used for load normalization (namely, maximum frequency and instructions per cycle).

Among all proposed approaches, IKS is the only officially accepted and mainstream solution, while GTS is currently discontinued in favor of a future Energy-Aware Scheduling (EAS) [Pitre 2015]. The major drawback of existing approaches is the use of an external entity to decide on the adequate frequency levels without any coordination with the scheduler. In fact, decoupling these domains may significantly impact the quality of scheduling decisions [Pitre 2014]. For these reasons, current developments are focused on integrating the DVFS subsystems in the kernel and extending the CFS functionality, such that all scheduling decisions are made from a consistent point of view [Pitre 2015]. However, incorporating this functionality is far from a trivial task, and the first-draft solutions are only expected in a couple of years [Pitre 2015].

The work proposed in this article aims at easing this burden by providing a general framework that relies on the original functionality of the default scheduling facilities in the current Linux-based systems (e.g., CFS, IKS, cpufreq) to extract the applicationspecific execution information (at runtime). This information is further used to combine the functionality of decoupled scheduling facilities and to explore new policies for future schedulers. As such, the work proposed herein tackles additional open research problems (as stated by the industry leaders [Rasmussen 2014]), such as (1) development of tools for evaluating the efficiency of scheduling approaches (Section 3), (2) definition of frequency-invariant metrics to describe the task load and resource utilization, and (3) scheduling of high-intensity tasks when the cores are fully utilized (Section 4).

As evidenced in PELT-based strategies, current techniques to describe the task load are solely based on the timing information available in the scheduler. Although important, this information is not sufficient to make the best scheduling decisions for any application [Rasmussen 2013; Pitre 2014]. For example, not all high-intensity tasks require high performance and/or they may depend on small tasks; thus, information about the application demands, importance, and dependencies is of the utmost importance. Furthermore, two tasks with different demands might spend a similar amount of time actively running on the allocated CPU and in the run queue; thus, they will be similarly characterized. This information does not encapsulate knowledge about the useful amount of performed work or how far the current task performance is from the actual application demands. Since all this information is property of the application, the current schedulers are unable to retrieve it. As a result, to derive more efficient scheduling policies, it is necessary to feed the system managers (scheduler) with information from the application itself, which is one of the key contributions of this work.

Introducing application-awareness to the scheduler is not an easy task, mainly due to (1) a large diversity of application characteristics and (2) the necessity of providing a unified metric to describe the application requirements and execution progress, as well as the capability of the underlying hardware to satisfy the application demands. For these reasons, we develop a flexible middleware solution that specifically focuses on iterative applications, that is, the applications that periodically perform a similar amount of work. In particular, by designating representative computational blocks, it is possible to introduce the self-reporting capabilities to these applications. As such, the task execution progress (i.e., task performance) can be described by the amount of representative computational blocks performed during a predefined time interval. 
Furthermore, instead of combining the in-scheduler timing information to derive the task load, by tracking the amount of performed computational blocks, it is possible to simultaneously distinguish between two different task performance values. First, by considering the total amount of time that the task spends in the run queue (while being coscheduled with other tasks on a single core), it is possible to express the observable task performance, that is, task performance as seen from the user space. Second, by considering the exact time intervals that the task spends in the active execution, it is possible to derive the maximum task performance at the current frequency of the processor (core). Accordingly, the application demands can be described as a desirable (minimum) task progress rate on a specific hardware (i.e., the performance target).

According to the state of the art [Brandenburg 2011; Zhu et al. 2015], any iterative process can be considered as a self-reporting application with a specific target performance, which includes rendering of images, video coding/decoding, data encryption/ decryption, or online modeling and real-time data processing (e.g., information tracking). Since the actual requirements and target performance are properties of the application, they must be encapsulated in the application itself (and communicated to the system managers): it can either be a fixed value dictated by what would be a pleasant user experience (e.g., a frame rate for animations) or be adjusted during execution.

With specified performance targets, one of the ultimate goals of the scheduler can be to reach the application target performance by changing the observable performance. This strategy provides an analytically tractable approach to satisfy the application demands by changing the task share in the scheduling period (nice value), processor/ core frequency, or both. The maximum task performance can be used as a relative measure of the core's compute capability, that is, the ability to satisfy the application demands. This mechanism also allows migrating high-intensity tasks to low-power clusters, whenever their performance targets can be met with the available frequency of "little" cores. Since the progress rate depends on the number of performed computational blocks per unit of time, it also intrinsically captures the effects of frequency scaling. While for compute-intensive tasks frequency scaling necessarily results in a logical performance change (higher progress rate at higher frequencies), the performance invariance of IO-bound tasks to the frequency scaling allows their agglomeration to "little" cores (independently of their PELT characterization).

It can be concluded that the application-guided task scheduling represents a very fruitful ground for future energy-aware schedulers, where many different scheduling policies can be derived. For this purpose, the framework proposed herein provides a standardized interface for enabling different types of parallel applications to report their performance. It also allows integrating different user-defined scheduling or optimization strategies (system managers) and enables dynamic adaptation according to the current state of the platform and application processing phases. This functionality is attained by relying on the feedback reported by the framework itself, that is, a highly accurate (near-scheduler) runtime monitoring subsystem.

\section{BEEPS: FRAMEWORK FOR APPLICATION-AWARE TASK MANAGEMENT}

The proposed framework relies on the original functionality of the default schedulers (namely, the CFS) and the DVFS mechanisms (e.g., cpufreq) to exploit the application requirements for energy-aware scheduling. This framework integrates many decoupled scheduling subsystems into a unified application-aware middleware located across both user and kernel space. As presented in Figure 1, the framework integrates a specifically developed Beeps subsystem, which consists of two main components: Beeps API and Beeps Driver (based on Taniça et al. [2014]). The Beeps API provides a standardized interface for enabling applications to report their performance, while the Beeps Driver provides highly accurate runtime system monitoring. By precisely assessing the 


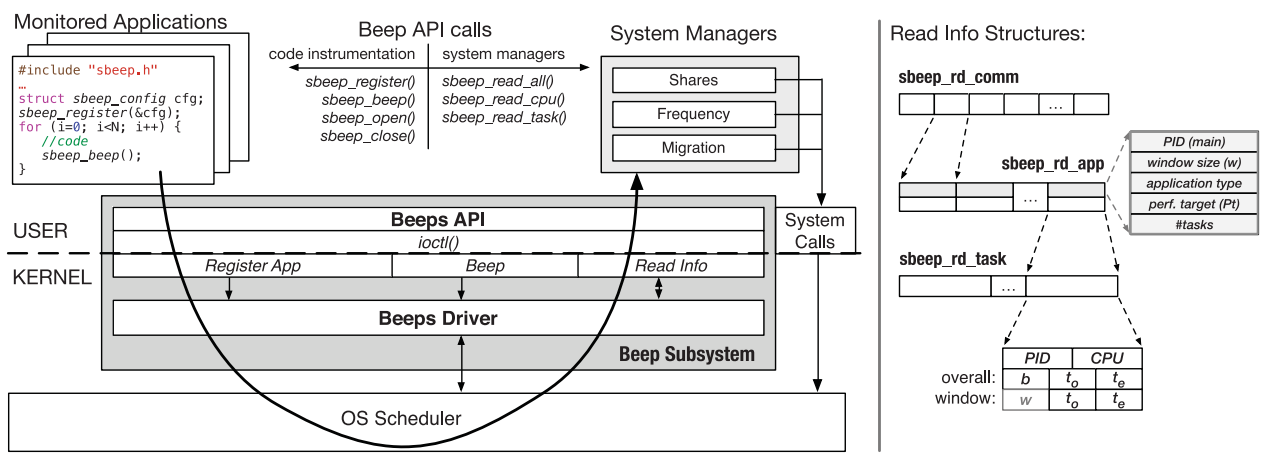

Fig. 1. Global overview of the proposed framework.

interaction between running applications/tasks and underlying hardware, the Beeps Driver also provides input data for a variety of user-defined system managers, which can be integrated in a plugin fashion.

\subsection{Beeps API}

As depicted in Figure 1, the developed Beeps API exports the framework's functionality to the user space through a small set of calls, each triggering a specific command in the underlying Beeps Driver by means of the ioctl() system calls. Hence, all target applications and system managers must integrate the proposed Beeps API, where the sbeep_open() and sbeep_close() should always be the first and the last call to the API, respectively. The main purpose of these calls is to create/destroy a file descriptor to the driver's device used by the other API calls (i.e., as a parameter to the ioctl calls).

On the application side, two API calls are provided, namely, sbeep_register() and sbeep_beep(). The sbeep_register call allows explicit registration of a parallel application to the monitoring driver, while the sbeep_beep enables application self-reporting via code instrumentation. For all registered applications, the underlying Beeps Driver automatically keeps record of all monitored tasks' performance. On the system manager side, the monitoring data can be retrieved through a set of sbeep_read_*() calls, namely, sbeep_read_all(),sbeep_read_cpu(), and sbeep_read_task(). The sbeep_read_all call allows retrieving the information about all currently registered applications, while sbeep_read_cpu and sbeep_read_task retrieve the information regarding the tasks running on a specific CPU core or even for just a specific task, respectively.

Once registered, a monitored application can report its performance by means of beeps, which are used as an application-specific progress measure. To introduce the self-reporting capabilities, the Beeps API provides a simple way of instrumenting the application code, where the representative computational blocks are designated with a single API call, namely, sbeep_beep(). By calling the sbeep_beep function, a performance event ( and communicated to the Beeps Driver via an ioctl system call. Afterward, the Beeps Driver automatically extracts the application performance information and decouples the performance contribution of each task of the target parallel application by relying on the kernel-space OS scheduling facilities. In detail, for applications with many parallel tasks, the beeps can be produced in many different ways depending on the structure and characteristics of the application. As such, in general, it is very difficult to model the performance of parallel workloads, that is, to analytically express the performance at the overall application level and at the level of individual tasks. For this purpose, 
we specifically focus on the two most common parallel application types, namely, type 1 applications, where each parallel task performs the representative computational block independently in parallel (i.e., each task produces its own beep), and type 2 applications, where all parallel tasks collaboratively contribute to a production of a single beep (e.g., when only the master task reports the performance and/or when all parallel tasks are invoked within the representative beep block).

As previously noted, prior to beep generation, an application must be registered into the framework by making the sbeep_register API call. The sbeep_register takes a single argument containing a configuration structure (shown as struct sbeep_config in Figure 1), which allows the configuration of the following parameters:

- performance window size $(w)$ - represents the size of a sliding window to report the performance (in number of beeps). The driver keeps a record and averages the target application's current performance based on the last $w$ beeps of each task.

-application type - designates the supported application type, that is, type 1 or type 2 applications. For type 1 applications, each task has its own performance window, which might not be synchronized with other tasks, and thus it may have a different time length. On other hand, for type 2 applications, the performance is obtained by propagating the main task's beeps through all the other application's tasks; that is, all tasks share the same beeps and monitoring window.

-inheritance flag - when enabled, all tasks descending from the registered task will be automatically registered and monitored by the Beeps Driver.

-target performance $\left(P_{T}\right)$ - allows specifying a desired progress rate of the application (in beeps per second). Since the plugged-in managers and the monitored applications do not directly communicate, the framework allows the applications to pass their target performance through the driver, which is communicated to the system managers whenever an sbeep_read_* call is performed.

The runtime performance information of the registered tasks can be retrieved by a user-space manager by using one of the sbeep_read_* calls. As presented in Figure 1 (see Read Info Structures), these calls take a pointer to an sbeep_rd_comm structure, which is automatically allocated and populated by the Beeps Driver. This structure contains an array of sbeep_rd_app structures, each with the general information for a specific application, namely, the process ID (PID) of the main task, the performance window size $(w)$, the previously configured target performance $\left(P_{T}\right)$ and application type, the number of tasks that constitute the application, and an array of sbeep_rd_task structures. The sbeep_rd_task structure contains the information of the performance for a single task, namely, its PID, the CPU core where the task is currently scheduled into, and a set of information since the beginning of the task execution and during a predefined window, that is, the total number of beeps (b), the amount of time spent in the run queue(s) $\left(t_{o}\right)$, and the amount of time spent during the actual task execution $\left(t_{e}\right)$.

\subsection{Beeps Driver}

The main functionality of the Beeps Driver is illustrated in Figure 2 with the examples of monitoring two different application types (each with two parallel tasks). Figure 2 also includes a set of events that trigger the main driver's functionality, in particular, a set of events corresponding to the Beeps API calls, that is, application registration $\left(B_{R}\right)$, beep production $\left(B_{B}\right)$, and retrieval of monitoring information $\left(B_{I}\right)$, as well as a set of OS scheduler events, that is, task fork $\left(S_{F}\right)$, switch $\left(S_{S}\right)$, migration $\left(S_{M}\right)$, and exit $\left(S_{E}\right)$.

As can be observed in Figure 2, for the type 1 application monitoring, at the beginning of the execution, task $A_{0}$ registers itself into the framework $\left(B_{R}\right)$ by using the 


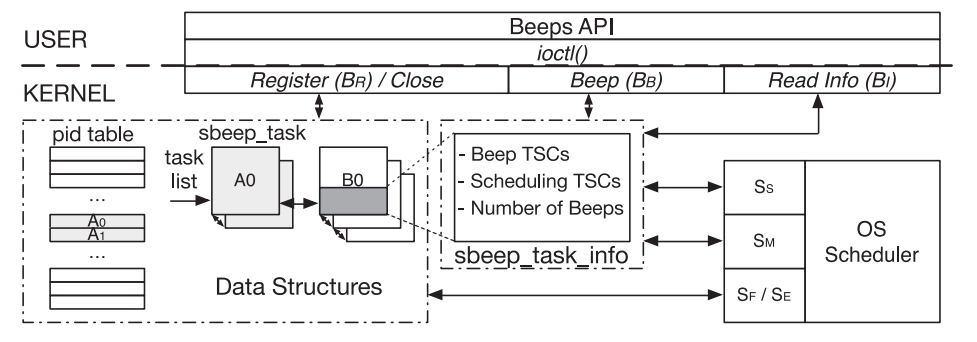

\section{Beeps API Events \\ $\mathrm{BR}$ - sbeep_register \\ BB - sbeep_beep? \\ $\mathrm{B} 1$ - sbeep_read_*0 \\ Scheduler Events}

SF - sched_process_fork 0

Ss - sched_process_switch

SE - sched_process_exit)

SM - sched_migrate_task0

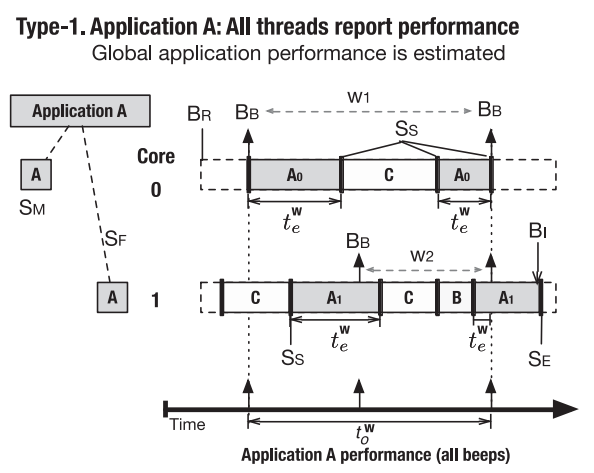

Type-2. Application C: One thread reports performance Individual thread performance is estimated

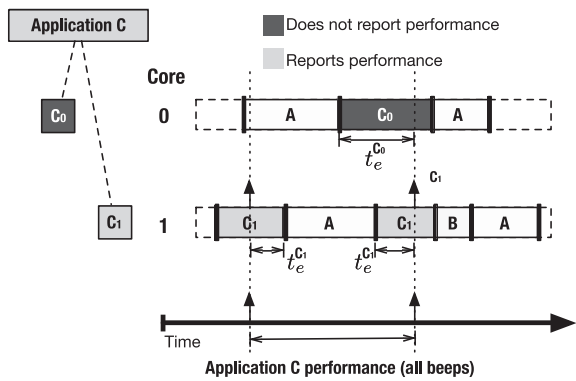

Fig. 2. Beeps Driver functionality and monitoring of different application types.

previously referred sbeep_register API call, with a window size of two and the inheritance flag enabled, and by selecting the type 1 application. Whenever a task is registered into the Beeps Driver, whether directly (through the API) or indirectly (through inheritance), the auxiliary data structures containing the monitoring configurations and for keeping track of the runtime performance are automatically allocated. To keep track of all registered applications (on a per-task basis) in an efficient way, two main data structures are used (1) a PID table and (2) a linked list tree holding a base structure for each monitored task, namely, sbeep_task. The PID table is used to reduce the overheads when referring to a specific task, that is, to avoid searching the complete list of tasks. Hence, the PID of a task corresponds to a single table entry, which contains a pointer to the corresponding kernel task structure (task_struct) in the task tree, if the task is registered (otherwise, the entry is defined as null).

In addition to the PID and a reference to the kernel task structure, each sbeep_task entry contains a sbeep_task_info structure (see Figure 2), where the obtained runtime performance parameters are kept. This structure mainly consists of an array of sbeep_beep_info structures, each containing the timestamps of the last $w$ beeps (where $w$ is the window size); the time interval and the execution time for each individual beep, that is, the time interval between two subsequent beeps $\left(t_{o}^{w}\right)$; and the time that the task was actually executing during that interval (represented as $t_{e}^{w}$ in Figure 2). In addition, this structure stores the total number of beeps $(b)$, time interval $\left(t_{o}\right)$ and actual execution time $\left(t_{e}\right)$ since the beginning of the task's execution, the current core where the task is running (or scheduled to run), and a set of auxiliary variables to keep track of the scheduling timestamps, thus allowing one to accurately track execution information for each monitored task.

Further on with the application's execution (see Figure 2), task $A_{0}$ forks another task, namely, $A_{1}$. Since the inheritance flag is enabled in the configuration structure, the task fork $\left(S_{F}\right)$ is detected by the Beeps Driver, which automatically registers task $A_{1}$ as a child of $A_{0}$. This functionality is attained by means of Linux kernel tracepoints, 
which are defined as available points in Linux code to which callback functions can be directly linked. Thus, whenever a specific part of Linux code is executed, the linked callback function will also be invoked. This allows inserting the pieces of code in several parts of Linux kernel code, as in specific scheduling functions, without the need for patching the kernel. In addition to forks (sched_process_fork, $S_{F}$ ), the driver is also able to detect several other OS scheduler events, namely, task terminations (sched_process_exit, $S_{E}$ ), task switches (sched_process_switch, $S_{S}$ ), and migrations across CPU cores (sched_migrate_task, $S_{M}$ ). A task switch (see $S_{S}$ in Figure 2) occurs whenever the OS scheduler substitutes a currently running task in a specific CPU core by another one, while a core migration $\left(S_{M}\right)$ occurs whenever the scheduler moves a task from one CPU core to another.

Whenever a scheduling event occurs, the Beeps Driver compares the target task PID against the PID table. If the task is registered (or its parent is registered with inheritance flag enabled), different actions take place depending on the event type. In case of a fork $\left(S_{F}\right)$, the process adheres to the previously described registration procedure. In case of a task switch $\left(S_{S}\right)$ or migration $\left(S_{M}\right)$, an update of the corresponding sbeep_task_info structure is performed, either to update the task's CPU or to record the scheduling timestamps. This allows not only detecting newly created tasks but also obtaining highly accurate per-task execution time and therefore accurate runtime performance information.

As can be observed in Figure 2, throughout the illustrated applications' execution excerpts, both tasks (within each application) perform several beeps $\left(B_{B}\right)$. Whenever the beep occurs (i.e., sbeep_beep API call), the corresponding task's sbeep_task_info structure is updated by incrementing the total number of beeps and the already referred time intervals and actual execution times (i.e., $b, t_{o}$, and $t_{e}$, respectively). The update of the sbeep_task_info structure must be an atomic operation; that is, it should be performed once at a time and without any interruption until completion. To guarantee this functionality, whenever a beep or a scheduling event is detected, both a synchronization mechanism and disabled preemption are needed. In fact, for the OS scheduling events, the preemption is automatically disabled, since the driver code is executed during the OS scheduler's execution. For these reasons, the sbeep_beep() API call was implemented as a system call. In this way, whenever a beep is performed within a task (user space), the beep execution is also performed from kernel space, thus allowing one to obtain the required environment for an atomic beep.

At the end of the execution depicted in Figure 2, an sbeep_read_* command is issued to the driver by a user-space manager ( $B_{I}$ event). Depending on the read call type, the Beeps Driver will retrieve the information regarding the registered tasks (on a per-application basis) and populate the provided sbeep_rd_comm structure with the available performance information, as previously described. For tasks $A_{0}$ and $A_{1}$, the last performance window is represented as $w_{1}$ and $w_{2}$, respectively. As such, the current performance of these tasks is obtained by using the observed time intervals $\left(t_{o}\right)$ and the actual execution times $\left(t_{e}\right)$ within the respective windows.

In particular, to attain a fine-grain execution control within the system managers, it is important to extract detailed per-application and per-task performance from the timing information gathered in the Beeps subsystem. However, as presented in Figure 2, depending on the type of monitored parallel applications, only one of these performance metrics can be physically captured, namely, per-task performance for the type 1 applications and the overall performance for the type 2 applications. As such, it is necessary to derive (by relying on the information from the Beeps Driver) the overall performance of type 1 applications and the performance of each task for type 2 applications.

As previously noted, the application performance is expressed as the total number of beeps reported by the complete process/thread hierarchy over a given period of time. 
Hence, for a type 1 application where $k$ parallel tasks independently report performance (see Figure 2), the observed application performance $\left(P_{O}\right)$ is defined as the sum of the reported beeps $\left(b_{i}^{(k)}\right)$ across all $k$ threads, such that

$$
P_{O}=\sum_{k} \sum_{i} b_{i}^{(k)} / t_{o}=\sum_{k} p_{o}^{(k)}
$$

where $p_{o}^{(k)}=\sum_{i} b_{i}^{(k)} / t_{0}$ is the observed performance for each task $k$ in a specific time interval $t_{o}$ (i.e., the performance directly retrievable from the Beeps Driver information).

As presented in Figure 2, for a type 2 application, where all tasks simultaneously contribute to a single performance milestone, that is, where a single task reports the beeps, only the overall observable application performance is explicitly defined, such that

$$
P_{O}=\sum_{i} b_{i} / t_{o}
$$

where $b_{i}$ represents the number of beeps generated by the master thread. In this case, the OS scheduling information provided by the Beeps Driver allows isolating per-task performance contributions $\left(p_{o}^{(k)}\right)$ by determining the exact time that each task spends in the execution state $\left(t_{e}\right)$. Hence, the observable per-task performance is estimated as

$$
p_{o}^{(k)}=P_{O} \times\left(t_{e}^{(k)} / t_{o}\right) .
$$

It should be noted that the usually observed task "execution" time $\left(t_{o}\right)$ only refers to the elapsed user-space time between two sbeep_beep calls, which is independent of the task state (see Figure 2). In contrast, $t_{e}^{(k)}$ refers to the actual time that a given task $k$ spent only in the execution state. Since CFS periodically preempts the execution of active tasks on a single core, such that only one task is allowed to execute at a time, $t_{o}$ can only match $t_{e}$ if a core is exclusively used by an always active task $k$.

It is worth emphasizing that the performance-oriented nature of the proposed framework is mainly driven by technical limitations of current Linux-based heterogeneous (embedded) systems to provide power consumption measures of adequate precision and monitoring granularity. In detail, to express power or energy efficiency on a per-task (or a per-application) basis, one has to obtain the exact power consumption during the time intervals monitored by the Beeps Driver, that is, both observable and actual time execution intervals. To accurately assess this information, power monitoring facilities with a very fine-grain measuring granularity are required at the level of logical cores. However, current platforms do not even provide power monitoring at the level of a single physical core. As such, one can only rely on the power consumption information at the overall CPU chip or a multicore cluster level. From this information, it is practically impossible to derive and distinguish the exact power contribution for each produced beep (either for a single task or for the overall application), especially in scenarios when several parallel applications are simultaneously coscheduled. However, for future platforms with adequate power monitoring facilities, integrating this functionality in the proposed framework is straightforward, since it only requires encapsulating the power consumption within each captured timing interval (as shown in Taniça et al. [2014]). In a similar sense, for systems providing access to performance counters, such information can also be easily integrated in the system, in order to provide per-task fine-grained performance characterization and phase identification.

The proposed framework represents a flexible middleware solution that allows unifying the functionality of decoupled system managers and testing different scheduling or optimization strategies based on the performance feedback reported by the framework itself. In Section 4, we exploit one of the possible ways of using this framework 
Table I. Summary of Used Parameters and Notations

\begin{tabular}{ll}
\hline Parameter & \\
\hline$b=\sum_{i} b_{i}$ & Number of performed beeps \\
$P_{T}$ & Application target performance \\
$P_{O} ; p_{O}$ & Observable performance (application; task) \\
$t_{O}$ & Observable task execution time (as seen from the user space) \\
$t_{e}$ & Actual (exact) task execution time on the allocated core \\
$s=t_{e} / t_{O} ; s_{T}$ & Task time share; target task share \\
$f$ & Operating frequency (core/cluster) \\
$I P B=I / b$ & Number of performed instructions (I) per beep \\
$C P I$ & Average number of clock cycles per instruction \\
$c=p_{O} / s_{O}=b / t_{e}$ & Performance parameter representing core capability to satisfy task demands \\
$\beta_{m \rightarrow n}=c_{n} / c_{m}$ & Performance ratio when migrating a task from core $m$ to core $n$ \\
$\alpha=P_{T} / P_{O}$ & Application performance scaling factor \\
$\left|1-p_{O} / p_{T}\right|$ & Normalized relative performance difference \\
$n^{(i)}$ & Nice level of task $i$ \\
$q=\alpha s_{O}$ & Per-task core utilization parameter (task share to fulfill the performance goal) \\
$U_{\mathcal{X}}=\sum_{i} q_{i}$ & Utilization of a core with assigned tasks $i$ (within cluster $\mathcal{X}$ ) \\
\hline
\end{tabular}

for soft real-time iterative applications with specified performance targets. However, the proposed framework is developed in a generic way; thus, it can be used to explore approaches that tackle different scheduling goals and criteria, such as power and temperature capping or task packing (core folding) concepts.

\section{SYSTEM MANAGERS FOR ATTAINING PERFORMANCE FAIRNESS}

The proposed set of system managers aims at controlling system resources, such as core frequency scaling and task-to-core allocations, in order to guarantee applications QoS while providing power savings. To attain this goal, the managers rely on the proposed Beeps framework to monitor not only the parent process but also all of its child threads and processes (which in Linux scheduling terminology are both indistinguishably called tasks), so as to provide support for single-threaded, multithreaded, and multiprocess applications. The proposed managers thus remain fundamentally different from the state of the art in the following aspects: (1) support the vast majority of parallel applications; (2) follow an online performance monitoring and modeling approach (see Section 4.1), supporting applications with variable workloads and with phase changes; and (3) do not follow a trial-and-error approach, which could often lead to erroneous decisions.

To ease understanding of the functionality of proposed managers in the following text, a brief description of used parameters and notations is provided in Table I.

\subsection{Task Performance Modeling}

To manage system resources, the proposed managers rely on a simple yet insightful model of task performance. Accordingly, the performance $p$ of a given task $k$ is given by the observed set of beeps $b$ over a wall time $t_{O}$, such that $p=b / t_{O}$. However, since in typical execution scenarios multiple tasks can be coscheduled to the same core, the actual performance is dependent only on the exact time $t_{e}$ that task $k$ was allowed to execute on the scheduled core or, equivalently, on the task time share $s=t_{e} / t_{O}$, thus allowing expressing the task performance as $p=b \times s / t_{e}$.

Naturally, the observed performance is affected by multiple factors, including the core operating frequency $f$ and the computational weight of the processing blocks, which in many applications may vary in real time. To account for the latter, we express the performance by considering the number of instructions $I$ required to execute the processing blocks and attain the observed beeps $b$, the average number of instructions 
to produce one beep $I P B=I / b$, and the average number of cycles per instruction $C P I$ :

$$
p=\frac{b \times s}{t_{e}}=\frac{I / I P B \times s}{I \times C P I / f}=\underbrace{\frac{1}{C P I \times I P B}}_{c} \times s \times f .
$$

This allows decoupling the task time share $s$ and the core operating frequency $f$ from the processing phase and workload variations, which are given by the ratio $c=1 /(C P I \times$ $I P B)$. However, performing a real-time estimation of the performance parameter $c$ is a nontrivial job, requiring input from both the application programmer and the processor. For computational platforms that have access to performance counters, IPB can be computed in real time by locally assuming a constant workload. For a task, the CPI can be estimated by considering core- and memory-related CPI and the ratio between core and memory operating frequency [Spiliopoulos et al. 2011; Su et al. 2014]. However, since this approach requires access to performance counters, which are not always available (as in the case of the considered Odroid XU+E board), adaptive modeling is employed to estimate the performance parameter $c$ in runtime. Hence, each time $t$ a beep is generated, the parameter $c$ is updated as

$$
c(t)=p_{O}(t) / s_{O}(t),
$$

where $p_{O}(t)$ and $s_{O}(t)$ are the observed task performance and time share, respectively, in the previous $W$ beeps. This allows estimating that in the period $[t, t+\Delta t]$, the task performance is given by

$$
p([t, t+\Delta t])=s([t, t+\Delta t]) \times c(t) \times f([t, t+\Delta t]) .
$$

In systems composed of multiple heterogeneous cores, multiple $c_{j}^{(i)}$ parameters are obtained and updated in runtime by relying on the Beeps subsystem. To abstract from phase changes and workload variations, whenever a task is migrated from core $m$ to core $n$, the performance ratio $\beta_{m \rightarrow n}^{(i)}=c_{n}^{(i)} / c_{m}^{(i)}\left(\beta_{n \rightarrow m}^{(i)}=1 / \beta_{m \rightarrow n}^{(i)}\right)$ is stored in the system by considering the parameters $c_{m}$ and $c_{n}$ before and after migration, respectively. Hence, whenever evaluating another core migration, one can estimate the corresponding $c_{j}$ value in the target core. Since at application start-up no prior information is available, an initial value of $\beta_{A 7 \rightarrow A 15}=2$ is set in the considered Exynous 5410 SoC, which is defined as a reasonable estimate according to our experimental validation and is supported by Colin et al. [2015] and Pricopi et al. [2013], where the actual value is shown to vary between 1 and 3, depending on the application characteristics. It should, however, be noted that, although a lookup table could be used to preinitialize the values according to the application characteristics (as in Cochran et al. [2011]), adaptive modeling is used to determine the exact value once a new beep is generated on the target core, in order to avoid errors due to phase changes.

\subsection{Algorithmic Overview of the Proposed Managers}

By relying on Equation (6), it is thus possible to perform a set of system-level optimizations that, by amending scheduling and frequency governor decisions, are able to modify $c, s$, and $f$ in order to provide application QoS while reducing the overall power consumption. Naturally, to attain this goal, the proposed managers rely on perapplication performance targets $P_{T}$, which can be directly or indirectly supplied by the user/programmer. Accordingly, assuming a set of parallel applications each spawning multiple tasks and a corresponding set of per-application performance targets, the functionality of the managers is the following: 


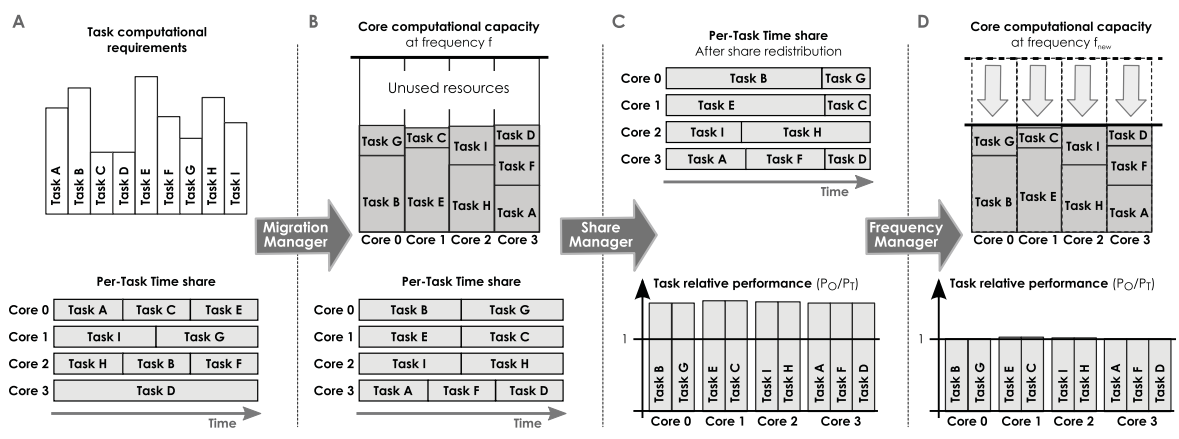

Fig. 3. Functionality overview of the proposed managers.

(1) As described in Section 3, by relying on the Beeps framework to monitor the complete execution hierarchy of each application and its corresponding overall performance $P_{O}$, obtain the performance contributions $p_{O}^{(i)}$ of each task $i$.

(2) Determine the per-task performance parameter $c^{(i)}$ using Equation (5).

(3) By evaluating the per-application performance error $E=\left|P_{O}-P_{T}\right|$, determine the application performance scaling factor $\alpha=P_{T} / P_{O}$, such that $\alpha P_{O}-P_{T}=0$. At the level of each task, this requires scaling the individual per-task execution times $t_{e}^{(i)}$, which is practically equivalent to scaling the observed performance $p_{O}^{(i)}$ by the same factor of $\alpha$. Accordingly, determine the per-task performance target $p_{T}^{(i)}$ as

$$
p_{T}^{(i)}=p_{O}^{(i)} \times \frac{P_{T}}{P_{O}} .
$$

(4) Allocate the tasks in the available cores in order to balance core workload, according to the predefined performance targets (as illustrated in Figure 3(B)); additionally, migrate tasks between heterogeneous cores in order to scale the per-task performance parameter $c$ (see Section 4.5 for additional details).

(5) As depicted in Figure 3(C) and detailed in Section 4.3, for each core $m$, optimize task time share $s$ in order to provide performance fairness at a per-core level; accordingly, for any tasks $k$ and $j$ scheduled to execute on the same core $m$, an equal relative performance is attained $\left(p_{O}^{(k)} / p_{T}^{(k)}=p_{O}^{(j)} / p_{T}^{(j)}\right)$.

(6) Scale the operating frequency of the cores to the minimum value $f$ that guarantees that all tasks attain the required performance level (as illustrated in Figure 3(D) and detailed in Section 4.4), that is, such that for any task $k$ on any core $m$ the relative performance is above $1\left(p_{O}^{(k)} / p_{T}^{(k)} \geq 1\right)$.

The following sections detail the functionality of the proposed share, frequency, and migration managers.

\subsection{Share Manager}

To achieve the performance target $\left(P_{T}\right)$ of a running parallel application, composed of $k$ parallel tasks, the observed application performance $\left(P_{O}\right)$ must be scaled by a factor of $\alpha=P_{T} / P_{O}$. Since different tasks may contribute differently to the global application performance, this requires scaling the exact time contribution $\left(t_{e}^{(k)}\right)$ of all application tasks by $\alpha$. To attain this goal at the level of each individual task, the proposed approach first sets the task performance target as $p_{T}^{(k)}=\alpha p_{o}^{(k)}$, so as to instruct scaling the task share $\left(s^{(k)}\right)$ by $\alpha$. However, according to current scheduler implementations, changing a task share causes inevitable adjustments to the shares of all other active tasks on 
the same core. For example, improving the task performance by increasing its share necessarily degrades the performance of all other tasks, because their shares must be reduced. Accordingly, the proposed managers adhere to a management concept that fosters performance fairness among running applications, that is, such that:

$$
\text { For any set of threads } i \text { an } j \text { scheduled to execute on core } m, \frac{p_{O}^{(i)}}{p_{T}^{(i)}}=\frac{p_{O}^{(j)}}{p_{T}^{(j)}} \text {. }
$$

Accordingly, the proposed share manager estimates the target share $\left(s_{T}^{(i)}\right)$ for each active task $i$ such that the task's relative performance difference $\left(\sum_{i}^{N}\left(1-p_{O}^{(i)} / p_{T}^{(i)}\right)\right)$ is minimized, while attaining performance fairness. By relying on the performance modeling described in Equation (4), on the core operating frequency $f$, and on the pertask performance parameters $c^{(i)}$, one obtains the following set of equations describing a per-core linear constrained optimization problem for a set of $N$ active tasks:

$$
\begin{aligned}
\min _{s_{T}^{(1)}, \ldots, s_{T}^{(N)}} & \sum_{i=1}^{N}\left(1-\frac{c^{(i)} s_{T}^{(i)}}{p_{T}^{(i)}}\right)^{2} \\
\text { s.t. } & \sum_{i=1}^{N} s_{T}^{(i)}=1 \quad \text { and } \quad \frac{c^{(i)} s_{T}^{(i)}}{p_{T}^{(i)}}=\frac{c^{(j)} s_{T}^{(j)}}{p_{T}^{(j)}} \text { for } i, j \in\{1, \ldots, N\},
\end{aligned}
$$

where Equation (9a) is used for normalized performance error minimization, the first condition in Equation (9b) ensures that the set of running tasks uses $100 \%$ of the core computational resources, and the second condition ensures performance fairness. By relying on the Karush-Kuhn-Tucker conditions [Kuhn and Tucker 1951], the share manager then obtains a unique solution for the per-task target shares $\left(s_{T}^{(i)}\right)$ in the real domain. Furthermore, based on the OS-defined share-to-nice conversion,

$$
s_{T}^{(i)}=\frac{1024 / 1.25^{n^{(i)}}}{\sum_{j} 1024 / 1.25^{n^{(j)}}},
$$

the shares are translated into integer task nice levels $\left(n^{(i)}\right)$ by solving

$$
n^{(i+1)}-n^{(i)}=\frac{\log s_{T}^{(i)}-\log s_{T}^{(i+1)}}{\log 1.25}, \forall i \in\{1, \ldots, N-1\},
$$

with an additional constraint that nice levels must be in a discrete interval [-20;19]. Finally, the proposed framework applies the calculated nice levels to the system.

\subsection{Frequency Manager}

The functionality of the frequency manager is tightly coupled with the decisions from the share manager. In detail, by equalizing the normalized performance among the running tasks, the share manager ensures that all tasks (in a particular core) attain a near-equal relative difference between the observed and target performance $\left(\left(p_{T}-\right.\right.$ $\left.\left.p_{O}\right) / p_{T}\right)$. The proposed frequency manager exploits this difference as a means for scaling the frequency such that the performance difference is minimized. For example, for a set of tasks with relative performance difference above the target, the frequency manager decreases the operating frequency, which allows reducing the performance difference while achieving significant power savings (see Figure 3(D)).

Depending on the target system, the proposed frequency manager is applied on a per-core or per-cluster level. In the former case, for each core $m$, the new frequency is 
calculated according to the relative performance of all tasks $i$ executing in that core:

$$
f_{\text {new }}^{(m)}=f_{\text {old }}^{(m)} \alpha_{\max } \quad \text { where } \quad \alpha_{\max }=\max _{i}\left\{p_{T}^{(i)} / p_{O}^{(i)}\right\},
$$

where $\alpha_{\max }$ is defined so as to overcome the limitations imposed by the finite number of nice levels, which may lead to slight differences between the share $s^{(i)}$ imposed via nice levels and the target share $s_{T}^{(i)}$. For systems where the frequency can only be set at a per-cluster level, a similar approach is adopted, by defining $\alpha_{\max }$ as the maximum ratio $p_{T}^{(i)} / p_{O}^{(i)}$ over all tasks scheduled to execute on any core within the cluster (although this may impose that tasks on a core $m$ perform over the target in order to allow tasks on the constrained core $n$ to execute on target, that is, such that for any task $p_{O}^{(i)} \geq p_{T}^{(i)}$ ).

Finally, it should be noted that, in modern heterogeneous systems, the frequency level is determined within a predefined range of discrete integer values, therefore requiring the use of the ceiling operator to round up the obtained frequency level. Naturally, this rounding operation limits a finer-grained control and leads to slight relative performance errors, as shown in Section 5.

\subsection{Intercluster and Intracluster Migration Manager}

The proposed migration manager aims at balancing the utilization across a set of cores and/or multicore clusters in heterogeneous systems. To achieve this functionality, it relies on per-task core utilization parameters $q^{(i)}$ :

$$
q^{(i)}=\frac{p_{T}^{(i)}}{c^{(i)} f}=\frac{P_{T}}{P_{O}} \frac{p_{O}^{(i)}}{c^{(i)} f}=\frac{P_{T}}{P_{O}} \frac{c^{(i)} s_{O}^{(i)} f}{c^{(i)} f}=\frac{P_{T}}{P_{O}} s_{O}^{(i)},
$$

which designate the share (or equivalently the $t_{e}$ ) required by each task $i$ to fulfill the performance goal at core $m$ while operating at frequency $f$. Thus, for a single core $m$ within cluster $\mathcal{X}$, its utilization $U_{\mathcal{X}}^{(m)}$ can be estimated as the sum of per-task parameters $q_{\mathcal{X}}^{(i)}$ across all assigned tasks $i$ :

$$
U_{\mathcal{X}}^{(m)}=\sum_{\substack{\text { All threads } i \\ \text { in core } m}} q_{\mathcal{X}}^{(i)}
$$

To balance the utilization among different cores at the level of a single multicore cluster $\mathcal{X}$, the migration manager relies on a queue with all tasks in the system (sorted in a nonincreasing order of their requirements $\left.q_{\mathcal{X}}^{(i)}\right)$ and iteratively allocates each task to the core with minimum utilization.

In heterogeneous embedded systems composed of multiple heterogeneous clusters, a similar procedure is achieved. In particular, for systems where only one cluster can be activated at a time, equilibrium is first achieved at the level of the active cluster $\mathcal{X}$ and then, by evaluating if a little energy-efficient cluster $\mathcal{Y}$ has enough processing power to cope with the required performance. Hence, by relying on the per-task performance scaling parameters $\beta_{\mathcal{X} \rightarrow \mathcal{Y}}^{(i)}$, one can determine the utilization of each core $m$ on the new cluster $\mathcal{Y}$ :

$$
U_{\mathcal{Y}}^{(m)}=\sum_{\substack{\text { All threads } i \\ \text { in core } m}} q_{\mathcal{Y}}^{(i)}=\sum_{\substack{\text { All threads } i \\ \text { in core } m}} \frac{p_{T}^{(i)}}{c_{\mathcal{Y}}^{(i)} f}=\frac{1}{f} \sum_{\substack{\text { All threads } i \\ \text { in core } m}} \frac{p_{T}^{(i)}}{c_{\mathcal{X}}^{(i)} \beta_{\mathcal{X} \rightarrow \mathcal{Y}}^{(i)}}
$$


Since core utilization must remain below $1\left(U_{\mathcal{Y}}^{(m)} \leq 1\right)$ in order to attain the required performance target, the following constraint must be fulfilled:

$$
f \geq \sum_{\substack{\text { All threads } i \\ \text { in core } m}} \frac{p_{T}^{(i)}}{c_{\mathcal{X}}^{(i)} \beta_{\mathcal{X} \rightarrow \mathcal{Y}}^{(i)}} .
$$

Accordingly, whenever the little cluster $\mathcal{Y}$ supports the required utilization in all cores, migration to little cores is performed, followed by frequency scaling and share manager invocation to rebalance the task shares. Otherwise, execution remains on a big cluster and frequency scaling is performed using a similar rule:

$$
f \geq \sum_{\substack{\text { All threads } i \\ \text { in core } m}} \frac{p_{T}^{(i)}}{c_{\mathcal{X}}^{(i)}} .
$$

In heterogeneous systems supporting the simultaneous activation of multiple clusters, the migration manager works by prioritizing the allocation of tasks to the little cores (using the set of parameters $q_{y}$ to estimate the utilization of the cores) and then distributing the remaining tasks to the big cores (using the set of $q_{\mathcal{X}}$ parameters). Finally, any core that remains unused, that is, without any active task, can be put under special-purpose idle states that provide power savings.

Finally, it should also be noted that the proposed managers can also benefit from power and energy models extracted in runtime from performance counters [Spiliopoulos et al. 2011; Su et al. 2014] whenever such infrastructure is provided (which is not in the considered Odroid XU+E board). Under such conditions, different optimization goals can be set. For example, by providing power and energy models, $P_{\mathcal{X}}^{(i)}(f)$ and $E_{\mathcal{X}}^{(i)}(f)$, respectively, for each active task $i$ and each core type $\mathcal{X}$, one can either scale the operating frequency to comply with strict power and/or energy constraints or adopt an energyefficient QoS execution by selecting (at a per-core or per-cluster level) the frequency $f$ that minimizes $E$ while still satisfying the application performance requirements, or by effectively allocating each task on the most energy-efficient core. Notwithstanding, additional research is required in order to generalize previous state-of-the-art techniques for counter-based power and energy models for any set of heterogeneous processors, which must not only provide accuracy at the level of each time slice but also be characterized by low computational requirements.

\section{EXPERIMENTAL EVALUATION}

To validate the proposed task management framework, a thorough experimental evaluation was conducted on an Odroid-XU+E development platform, powered by a Samsung Exynos 5410 SoC with a four-core in-order Cortex-A7 cluster and a four-core out-oforder Cortex-A15 cluster. The platform supports cluster-migration mode only, resulting in the activation of either the A7 cluster or the A15 cluster, as follows: for a virtual operating frequency in the range of $[250,600] \mathrm{MHz}$, the A7 cluster is activated with a real operating frequency of twice the virtual one; for a virtual operating frequency in the range of $[800,1600] \mathrm{MHz}$, the Cortex-A15 cluster is activated and operated at the same real frequency as the virtual one. As such, the chosen experimental setup represents one of the most challenging scenarios to evaluate the efficiency of the proposed framework and system managers. The considered platform also integrates a power measurement IC, accessible by software, which allows for accurate runtime energy monitoring, with values updated at each $300 \mathrm{~ms}$. 
Table II. Benchmarks Used for Experimental Validation of the Proposed Framework

\begin{tabular}{|c|c|c|c|}
\hline & \multirow{2}{*}{$\begin{array}{l}\text { Performance } \\
\text { Reporting }\end{array}$} & \multirow{2}{*}{$\begin{array}{l}\text { Max. Performance } \\
\text { (with } 1 \text { thread) }\end{array}$} \\
\hline \multicolumn{2}{|c|}{$\begin{array}{l}\text { SPEC CPU2006 (single-threaded applications) } \\
\text { Application Input }\end{array}$} & & \\
\hline h264 & train & 1 beep $=1$ frame & $0.55 \mathrm{beeps} / \mathrm{s}$ \\
\hline HMMER & train & 1 beep $=256$ samples & $2.55 \mathrm{beeps} / \mathrm{s}$ \\
\hline LBM & ref (max. 300 steps) & 1 beep $=1$ time step & $1.35 \mathrm{beeps} / \mathrm{s}$ \\
\hline \multicolumn{2}{|c|}{ PARSEC (multi-threaded applications) } & Performance & Max. Performance \\
\hline Application & Input & Reporting & (with 4 threads) \\
\hline Swaptions $^{(a)}$ & Swaptions $=128$, simulations $=40,000$ & 1 beep $=1$ swaption & $1.0 \mathrm{beeps} / \mathrm{s}$ \\
\hline BlackScholes $^{(a)}$ & in_10M.txt & 1 beep $=25,000$ options & $157 \mathrm{beeps} / \mathrm{s}$ \\
\hline Fluidanimate ${ }^{(a)}$ & in_200k.fluid, frames $=250$ & 1 beep $=1$ frame & $2.5 \mathrm{beeps} / \mathrm{s}$ \\
\hline $\mathrm{x} 264^{(b)}$ & sintel_trailer_2k_480p_24.y4m & 1 beep $=1$ frame & $26.2 \mathrm{beeps} / \mathrm{s}$ \\
\hline
\end{tabular}

(a) Each thread reports its own performance.

${ }^{(b)}$ One thread reports the overall application performance.

\subsection{Benchmark Applications}

To analyze and evaluate the functionality of the proposed framework, a set of singleand multithreaded workloads was selected from the SPEC CPU2006 and PARSEC benchmark suites, considering both the representation of real QoS problems [Misailovic et al. 2010] and its use in the related work [Hoffmann et al. 2013; Imes et al. 2015; Muthukaruppan et al. 2014; Vega et al. 2013b], namely, h264, HMMER, and LBM (from SPEC CPU2006), and blackscholes, fluidanimate, swaptions, and x264 (from PARSEC). For example, $h 264$ and $x 264$ are video-coding applications whose QoS targets are related with the encoding and whose computational complexity is highly dependent on both the input video and the encoding parameters; streamcluster is an iterative application for online clustering, for which QoS can be either defined by the rate at which new data is received or programmer defined by using clustering-specific metrics to determine the number of iterations in order to attain good clustering quality [Misailovic et al. 2010]; swaptions and blackscholes are iterative applications that evaluate the price of options, for which QoS can be measured as the scaled difference of the price options [Misailovic et al. 2010]. As can be perceived, different applicationspecific QoS metrics could, and in most cases have to, be defined. However, to abstract from application-specific QoS definitions, in the following set of experimental results the application targets are varied according to the system computational requirements.

To allow for performance reporting, the applications were instrumented using the Beeps API, including the main loop where a call to sbeep_beep() was inserted at the beginning of each iteration. The resulting performance reports are presented in the third column of Table II. As a result of such changes, different performance reports are attained. For single-threaded applications (namely, those taken from the SPEC CPU benchmark suite), both thread and application performance are directly attained from the reported beeps. For multithreaded applications, one of the following cases may occur: (1) each thread reports its own performance (as in the case of blackscholes, fluidanimate, and swaptions) and global application performance is estimated by relying on Equation (1), or (2) one thread reports the global application performance (as is the case of $x 264$ ), requiring the computation of Equation (3) to estimate per-thread performance.

To further characterize the considered benchmarks, the application's maximum performance was determined by executing it in the A15 cluster at the maximum operating frequency $(1.6 \mathrm{GHz})$. The application's maximum performance is then determined by computing the ratio between the total number of beeps and the execution time between 

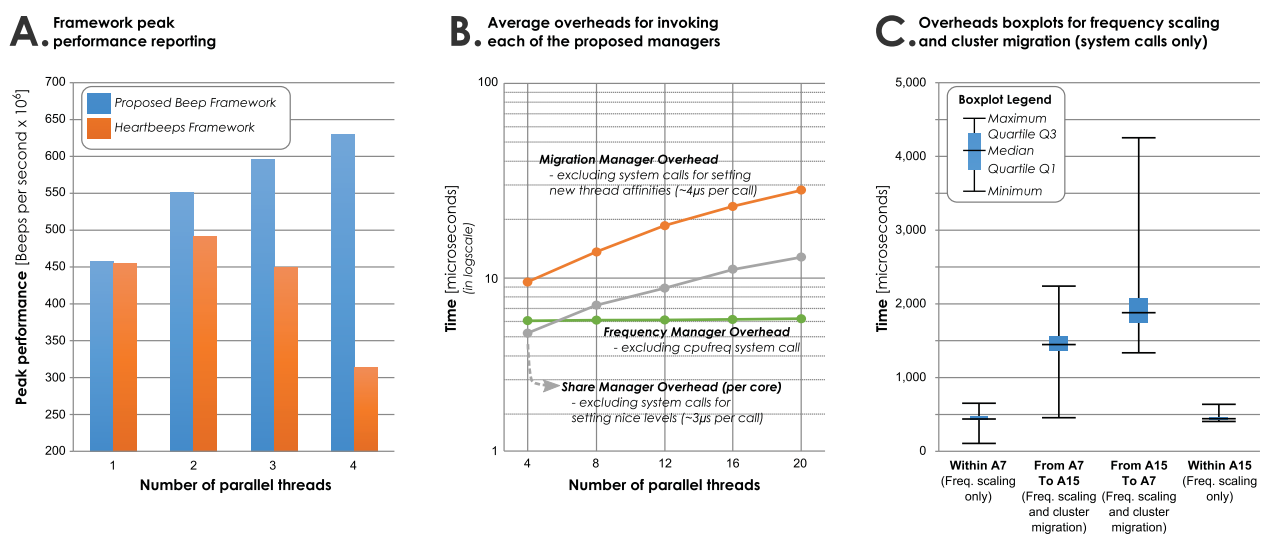

Fig. 4. Overhead analysis of the proposed Beeps framework.

the first and the last beep (thus excluding the time for application start and finish). The obtained results are also presented in Table II.

\subsection{Framework Overheads}

In order to experimentally assess the capabilities of the proposed Beeps framework and of the devised system managers, the imposed overheads are first assessed by relying on a set of synthetic benchmarks. The first benchmark assesses the maximum performance that can be reported by the Beeps framework using the A15 cluster of cores while operating at the maximum frequency $(1.6 \mathrm{GHz})$. Hence, the benchmark spawns a set of $x \in\{1,2,3,4\}$ threads, each consisting of a single loop performing 10 million calls to the sbeep_beep function, and thus generating an equivalent number of beeps (no other operation is performed). Moreover, to validate the obtained results, an equivalent benchmark was devised to stress the Heartbeats framework [Hoffmann et al. 2010] and attain its maximum reporting performance. As can be concluded by the results depicted in Figure 4(A), the Beeps framework allows for higher performance reporting. For example, when using four threads (one per core), with the Beeps framework a performance of $630 \mathrm{k}$ beeps/s (latency of $1.59 \mu \mathrm{s}$ per beep) is attained, whereas the Heartbeats framework is limited at $314 \mathrm{k}$ beeps/s (latency of $3.19 \mu \mathrm{s}$ per beep). These results are a direct consequence of the multiple optimizations performed in the Beeps framework (see Section 3) and of the synchronization procedures required by the Heartbeats framework implementation [Sironi et al. 2012].

The second benchmark application was devised to evaluate the execution overheads for the proposed managers while executing a set of $y=\{1,2,3,4,5\}$ swaptions applications (each with four threads). In order to fully stress the system, variable application targets were defined to guarantee evaluating different system configurations and different management decisions. The average execution time (excluding system calls) for executing each manager is presented in Figure 4(B). As can be observed, when managing 20 threads (five per core), there is a reduced overhead of less than $12 \mu \mathrm{s}$ (per core) and $7 \mu \mathrm{s}$ (per cluster) for the share and frequency managers, respectively, whereas a $30 \mu \mathrm{s}$ overhead is observed for the migration manager, which requires computing and sorting the per-task utilization parameters $q$, distributing the tasks along the cores, and verifying if execution should be migrated to another cluster.

Finally, additional benchmarks were devised to assess the overheads due to the system calls that implement the managers' decisions, such as for setting nice levels ( $3 \mu \mathrm{s}$ per task, on average) and task affinities ( $4 \mu \mathrm{s}$ per task, on average), and especially 


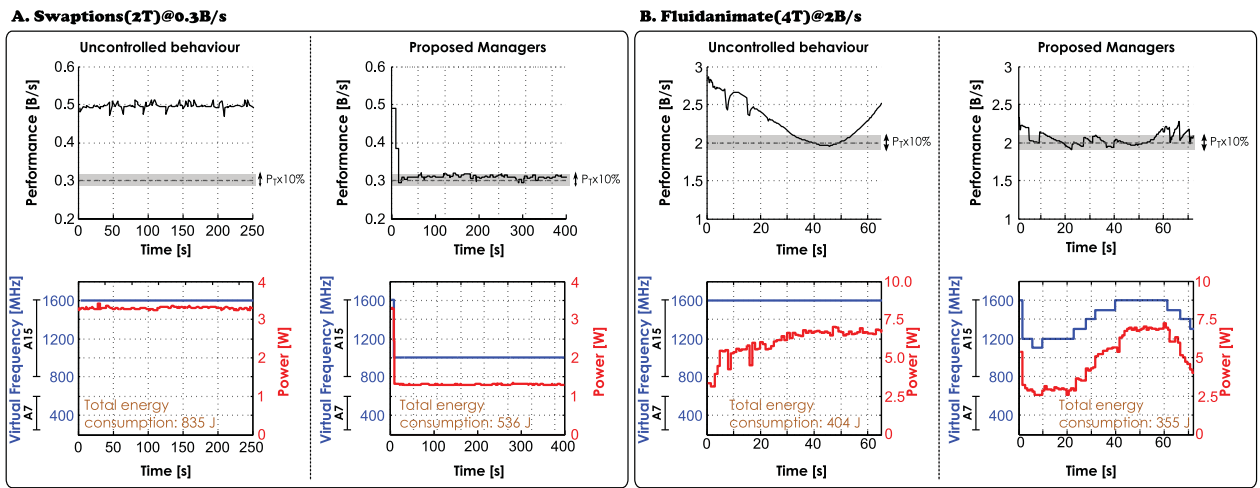

Fig. 5. Comparison of the proposed Beeps framework and managers with the default system scheduler when using the interactive governor.

for frequency scaling and for cluster migration, which represent the most expensive operations (see Figure 4(C)). In particular, while intracluster frequency scaling imposes an overhead of $440 \mu \mathrm{s}$ (median time), performing cluster migration typically requires around $1.5 \mathrm{~ms}$. Hence, to mitigate such overheads, in the following set of experimental results, the share manager is activated at a $10 \mathrm{~ms}$ time interval, updating shares at a per-core level if new performance information is present. The frequency and migration managers are called after five and 15 updates to the share manager, respectively.

\subsection{Managers' Global Behavior}

By relying on the previously noted managers' configuration, a set of benchmarks is used to thoroughly evaluate the system. In particular, we start with a set of simple case studies where a single application is set to run on the Odroid XU+E board, so as to avoid overloading the system. Hence, the system behavior is first analyzed using swaptions (two threads) and then using fluidanimate (four threads), with performance targets of 0.3 and 2.0 beeps/second, respectively. By analyzing the results shown in Figure 5, it can be concluded that, in both cases, the default system behavior (using the interactive governor) is to immediately migrate the execution to the A15 cluster and set it to operate at the maximum frequency. Accordingly, while it is able to operate over the target, it forces higher power and energy consumption.

On the other hand, the proposed managers are able to attain the performance target and also save a significant amount of energy. In detail, in case A (or case B), when the two swaptions (or four fluidanimate) threads are launched, the migration manager distributes them among two (or four) cores, so as to lower the per-core workload and allow operating at lower frequencies (the share manager has no noticeable impact in the results since there is a single task per core in each case). Hence, after receiving two sets of beeps ( $t=4.8 \mathrm{~s}$ for swaptions and $t=1.8 \mathrm{~s}$ for fluidanimate), the frequency manager activates and lowers the operating frequency to $1 \mathrm{GHz}(1.1 \mathrm{GHz}$ for case study B). Since the swaptions benchmark has a performance variation of at most $8 \%$ when running on the A15 cluster @ $1 \mathrm{GHz}$, and the obtained performance is always above the target, no other frequency scaling operation is performed until the end of execution. However, a different behavior is observed for case B. Since the fluidanimate computational requirements vary significantly during execution (as is clearly shown by the uncontrolled behavior in Figure 5(B)), the frequency manager is forced to adjust in runtime the operating frequency in order to attain the performance target. Accordingly, it is able to keep the application performance within a $10 \%$ interval of the target performance while still providing the means to save power. 


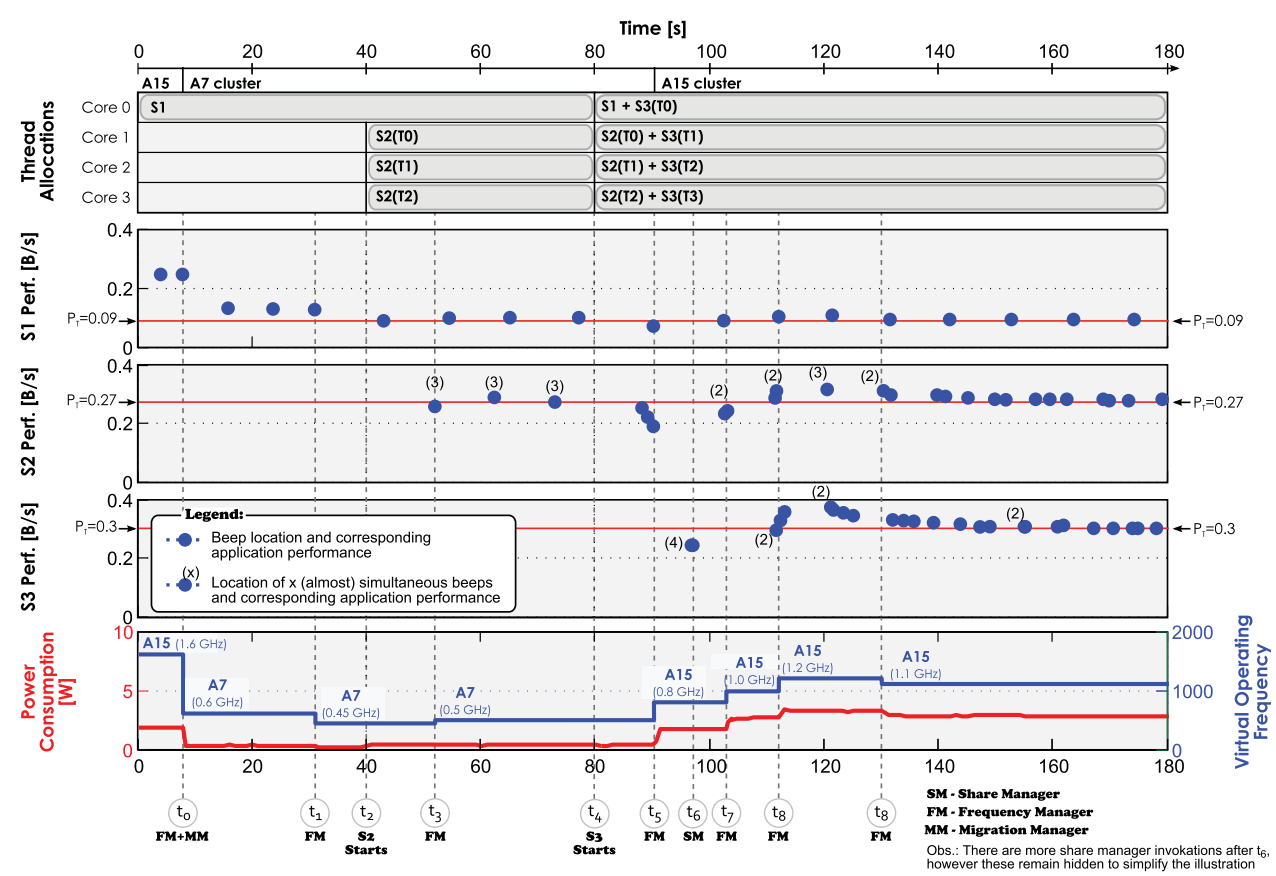

Fig. 6. Detailed behavior of the Beeps framework and proposed managers.

To thoroughly demonstrate the behavior of the proposed managers with a real example, a new benchmark was devised consisting of three applications, starting at different time instants, namely, $t=0 \mathrm{~s}, t=40 \mathrm{~s}$, and $t=80 \mathrm{~s}$. To allow graphically illustrating the system and managers' behavior, we rely on the swaptions application because of its low beep rate. However, each swaptions is set to use a different number of threads and to attain a different performance goal. In particular, the first swaptions (S1) is set to attain a target performance of $0.09 \mathrm{~B} / \mathrm{s}$ using a single thread; the second swaptions (S2) launches three threads but requires a performance of $0.27 \mathrm{~B} / \mathrm{s}$; finally, the last swaptions (S3) is required to attain a performance of $0.3 \mathrm{~B} / \mathrm{s}$ using four threads.

The obtained results, including the execution temporal diagram and the managers' runtime behavior, are illustrated in Figure 6. As can be observed, since the system is initially set to operate on the A15 cluster @1.6GHz, the performance of S1 is initially over the target. Accordingly, after receiving two sets of beeps from $\mathrm{S} 1$ ( $t_{0}$ in Figure 6), the managers compute the utilization parameter for core $0\left(U_{A 15}^{(0)}\right)$ and determine that the execution can be migrated to the A7 cluster. Since there is no previous information about swaptions performance on the $\mathrm{A} 7$ cluster, it uses the static relative performance of $\beta_{A 7 \rightarrow A 15}=2$ to estimate $p_{A 7}=p_{A 15} / \beta_{A 7 \rightarrow A 15}$. However, since the actual value is $\beta_{A 7 \rightarrow A 15}=1.46$, the estimated frequency remains above the target. Accordingly, after receiving two additional beeps on the A7 cluster $\left(t_{1}\right.$ in Figure 6$)$, the frequency manager scales the operating frequency to $900 \mathrm{MHz}$ (virtual frequency of $450 \mathrm{MHz}$ ) and reaches the requested target performance.

At time $t_{2}$, the second swaptions application (S2) starts executing. Accordingly, the migration manager evenly distributes the tasks along the remaining cores $(1,2$, and 3 ), hence giving each active task exclusivity over a different core. Notwithstanding, when the first performance reports from S2 are given $\left(t_{3}\right)$, the managers detect that the 
performance is below the target. Accordingly, the frequency manager scales operating frequency up, such that the desired targets for both S1 and S2 are reached.

When the third swaptions application (S3) starts $\left(t_{4}\right)$, the system manager attempts again to evenly distribute the workload, allocating one S3 thread per core. Naturally, this requires the previously running threads to lose exclusive rights over its core, which leads to a decrease in the performance of both S1 and S2. To compensate for such performance loss $\left(t_{5}\right)$, the managers attempt to increase the frequency, discovering that the application performance goals cannot be attained at the maximum frequency of the A7 cluster, that is, $f_{\text {new }}=f_{\text {old }} \times \max \left\{p_{T}^{(i)} / p_{O}^{(i)}\right\}>1.2 \mathrm{GHz}$. Accordingly, execution is migrated to the A15 cluster @ 800MHz, where the target frequency is determined by relying on the previously obtained value of $\beta_{A 7 \rightarrow A 15}=1.46$ for $\mathrm{S} 1$, and the default value of $\beta_{A 7 \rightarrow A 15}=2$ for all S2 threads (the actual per-task value is determined after receiving two beeps from each $\mathrm{S} 2$ thread). This allows estimating the per-task performances $p_{A 15}^{(i)}=p_{A 7}^{(i)} / \beta A 7 \rightarrow A 15$, which are then used to determine the operating frequency as $f_{\text {new }}=f_{\text {old }} \times \max \left\{p_{T}^{(i)} / p_{A 15}^{(i)}\right\}$.

Although until $t_{6}$ there is no effective contribution of the share manager, it has a profound impact on the system after the first performance information is received from the S3 threads. In particular, from this point onward, the share manager is invoked multiple times (at each new beep arrival) in order to balance the per-task performance error $\left(\left(p_{O}^{(i)}-p_{T}^{(i)}\right) / p_{T}^{(i)}\right)$ and attain performance fairness at a per-core level. Hence, due to its effective contribution, the proposed managers are able to discover a solution that guarantees performance fairness to all applications even though there are small workload changes throughout the execution, which are compensated by nice level adaptations and frequency scaling operations (see time instants $t_{7}, t_{8}$, and $t_{9}$ ).

\subsection{Managers Evaluation under Complex Workloads}

While the experimental results in Figure 6 already provide important insights into the proposed framework, a broader selection of results was taken by considering different and combinations of representative real workloads.

To highlight the contributions of the proposed work, the obtained results are compared against the default system scheduler using three different cpufreq governors, namely, interactive, ondemand, and conservative. In particular, ondemand is commonly used by smartphone manufacturers because it aims at providing a smooth performance by scaling the frequency up whenever a threshold load is detected by any running application. The conservative and interactive governors have a similar behavior to ondemand. However, the conservative governor leads to smoother changes in operating frequency, so as to favor battery-powered environments. The interactive governor favors latency-sensitive, interactive workloads, by checking the CPU load immediately after coming out of idle mode (e.g., due to the launch of an application), instead of simply sampling it at regular time intervals.

In particular, workload A represents the concurrent execution of two stable swaptions applications with a moderate performance target. As can be observed from the results in Figure 7, the proposed framework allows balancing the application's performance with significant energy savings by mainly running the tasks on the A7 cluster.

Workload B presents a challenging problem for the migration managers by forcing the distribution of six threads over four cores. Accordingly, in order to balance the per-core workload, the migration manager is forced to search for a solution in a discrete and finite set of possible task-to-core allocations where optimality is nonexistent. Nevertheless, the system successfully achieves near-target performance. Moreover, by allowing the execution to be migrated to the energy-efficient A7 cluster, it allows for a 


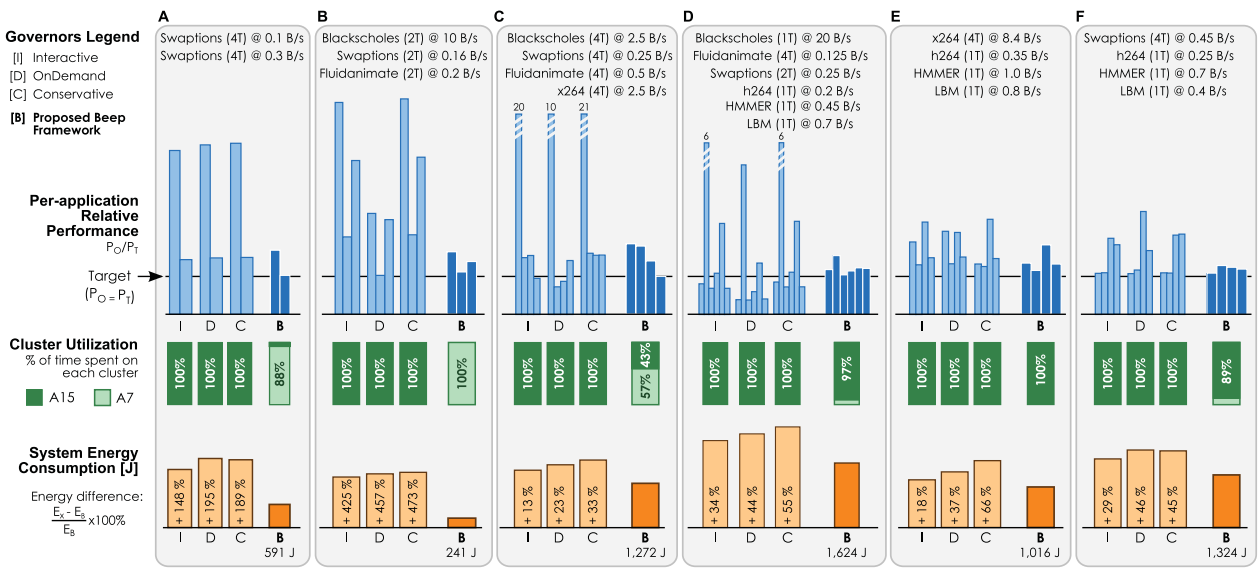

Fig. 7. Comparison of the proposed Beeps framework (B) with the default system scheduler when using the interactive (I), on-demand (D) or conservative (C) governors. Each benchmark (A-F) corresponds to the concurrent execution of a set of applications, referred to as application name (number of threads) @ target performance.

substantial reduction in energy consumption. In comparison, the default system scheduler, allied with the considered governors, requires over $400 \%$ more energy by not using the available opportunities to migrate execution to the energy-efficient A7 cluster.

Example $\mathrm{C}$ represents a mixed workload incorporating the $x 264$ encoder. Due to the nature of the $x 264$ algorithm, the framework is required to handle frequent phase changes, related with the encoding of different I, P, and B frames, each requiring a different number of operations to achieve one beep. Notwithstanding, the managers are able provide QoS while attaining significant energy savings.

Case studies D through $\mathrm{F}$ feature not only PARSEC benchmarks but also singlethreaded benchmarks from the SPEC CPU2006 suite. Moreover, to challenge the proposed management framework and to decrease the potential to exploit the energy efficiency of the A7 cluster, higher performance targets were set. Nonetheless, significant energy savings were still obtained by relying on the frequency manager and by exploiting the few remaining opportunities to run on the A7 cluster. Moreover, by relying on the default scheduler and on the cpufreq governors, in benchmark $\mathrm{D}$, the system was unable to satisfy the performance goals of blackscholes, swaptions, and LBM. This is a consequence of the default time fairness behavior, which aims at equally distributing core execution time among all the applications. By relying on the proposed system, all performance targets were met, guaranteeing the system QoS.

\section{CONCLUSIONS}

In this article, a general framework was proposed for efficient application-aware task management on heterogeneous embedded systems with several multicore clusters. The proposed framework tackles the fundamental OS task management aspects by integrating the specifically developed Beeps subsystem for highly accurate runtime performance monitoring and performance self-reporting for different parallel and iterative applications. Furthermore, it allows incorporating a set of system managers for efficient resource utilization, frequency scaling, and task migration at intra- and intercluster levels. Experimental results show that the proposed framework and system managers were capable of complying with the specified soft real-time performance targets for a set of iterative real-world SPEC CPU2006 and PARSEC benchmarks. When 
compared with the default system scheduling approaches and strategies, significant energy savings were also achieved in a state-of-the-art embedded platform.

\section{REFERENCES}

Björn B. Brandenburg. 2011. Scheduling and Locking in Multiprocessor Real-time Operating Systems. Ph.D. Dissertation. University of North Carolina at Chapel Hill.

John M. Calandrino, Hennadiy Leontyev, Aaron Block, Uma Maheswari C. Devi, and James H. Anderson. 2006. LITMUS: Testbed for empirically comparing real-time multiprocessor schedulers. In Proceedings of the IEEE International Real-Time Systems Symposium (RTSS'06). IEEE, 137-149. DOI : http://dx.doi.org/ 10.1109/RTSS.2006.27

Ryan Cochran, Can Hankendi, Ayse K. Coskun, and Sherief Reda. 2011. Pack \& Cap: Adaptive DVFS and thread packing under power caps. In Proceedings of the Annual IEEE/ACM International Symposium on Microarchitecture (MICRO-47'11). ACM, New York, NY, 175-185. DOI : http://dx.doi.org/10.1145/ 2155620.2155641

Alexei Colin, Arvind Kandhalu, and Ragunathan Raj Rajkumar. 2015. Energy-efficient allocation of real-time applications onto single-ISA heterogeneous multi-core processors. Journal of Signal Processing Systems (2015), 1-20. DOI : http://dx.doi.org/10.1007/s11265-015-0987-3

Jonathan Corbet. 2013. Power-Aware Scheduling Meets a Line in the Sand. http://lwn.net/Articles/552885/. (Jun 2013).

Hadi Esmaeilzadeh, Emily Blem, Renee St. Amant, Karthikeyan Sankaralingam, and Doug Burger. 2011. Dark silicon and the end of multicore scaling. In Proceedings of the International Symposium on Computer Architecture (ISCA'11). IEEE, 365-376.

Francisco Gaspar, Aleksandar Ilic, Pedro Tomas, and Leonel Sousa. 2014. Performance-aware task management and frequency scaling in embedded systems. In Proceedings of the International Symposium on Computer Architecture and High Performance Computing (SBAC-PAD'14). IEEE, 65-72.

Henry Hoffmann, Jonathan Eastep, Marco D. Santambrogio, Jason E. Miller, and Anant Agarwal. 2010. Application heartbeats for software performance and health. In Proceedings of the SIGPLAN Annual Symposium on Principles and Practice of Parallel Programming (PPoPP'10), Vol. 45. ACM, New York, NY, 347-348.

Henry Hoffmann, Martina Maggio, Marco D. Santambrogio, Alberto Leva, and Abhishek Agarwal. 2013. A generalized software framework for accurate and efficient management of performance goals. In Proceedings of the International Conference on Embedded Software (EMSOFT'13). IEEE, 1-10.

Connor Imes and Henry Hoffmann. 2015. Minimizing energy under performance constraints on embedded platforms: Resource allocation heuristics for homogeneous and single-ISA heterogeneous multi-cores. SIGBED Review 11, 4 (2015), 49-54. DOI : http://dx.doi.org/10.1145/2724942.2724950

Connor Imes, David H. K. Kim, Martina Maggio, and Henry Hoffmann. 2015. POET: A portable approach to minimizing energy under soft real-time constraints. In Proceedings of the Real-Time and Embedded Technology and Applications Symposium (RTAS'15). IEEE, 75-86.

Brian Jeff. 2013. big.LITTLE Technology Moves Towards Fully Heterogeneous Global Task Scheduling. ARM Ltd. White Paper.

H. W. Kuhn and A. W. Tucker. 1951. Nonlinear programming. In Proceedings of the 2nd Berkeley Symposium on Mathematical Statistics and Probability. University of California Press, Berkeley, CA, 481-492.

Kai Ma, Xue Li, Ming Chen, and Xiaorui Wang. 2011. Scalable power control for many-core architectures running multi-threaded applications. In Proceedings of the International Symposium on Computer Architecture (ISCA'11), Vol. 39. ACM, 449-460.

Sasa Misailovic, Stelios Sidiroglou, Henry Hoffmann, and Martin Rinard. 2010. Quality of service profiling. In Proceedings of the ACM/IEEE International Conference on Software Engineering (ICSE'10). ACM, 25-34.

Steve Muckle. 2014. A QuIC Take on Energy-Aware Scheduling. Linaro Connect USA (LCU). (2014).

Thannirmalai S. Muthukaruppan, Anuj Pathania, and Tulika Mitra. 2014. Price theory based power management for heterogeneous multi-cores. In Proceedings of the International Conference on Architectural Support for Programming Languages and Operating Systems (ASPLOS'14). ACM, 161-176.

Thannirmalai S. Muthukaruppan, Mihai Pricopi, Vanchinathan Venkataramani, Tulika Mitra, and Sanjay Vishin. 2013. Hierarchical power management for asymmetric multi-core in dark silicon era. In Proceedings of the Design Automation Conference (DAC'13). ACM, 174.

Pengcheng Nie and Zhenhua Duan. 2012. Efficient and scalable scheduling for performance heterogeneous multicore systems. Journal of Parallel and Distributed Computing 72, 3 (2012), 353-361. DOI:http:// dx.doi.org/10.1016/j.jpdc.2011.12.005 
Nicolas Pitre. 2014. Teaching the Scheduler About Power Management. Retrieved from http://wn.net/ Articles/602479/.

Nicolas Pitre. 2015. The Road to Energy-Aware Scheduling. Linaros Executive Member Update. Retrieved from http://www.linaro.org/blog/core-dump/road-energy-aware-scheduling/.

Mathieu Poirier. 2013. In Kernel Switcher: A solution to support ARM's new big.LITTLE technology. In Embedded Linux Conference (ELC'13).

Mathieu Poirier. 2014. GTS - A solution to support ARM's big.LITTLE technology. In Linaro Connect Asia (LCA'14).

Mihai Pricopi, Thannirmalai S. Muthukaruppan, Vanchinathan Venkataramani, Tulika Mitra, and Sanjay Vishin. 2013. Power-performance modeling on asymmetric multi-cores. In Proceedings of the International Conference on Compilers, Architecture and Synthesis for Embedded Systems (CASES'13). IEEE, $1-10$.

Morten Rasmussen. 2013. Using task load tracking to improve kernel scheduler load balancing. In Linux Foundation Collaboration Summit (LFCS'13).

Morten Rasmussen. 2014. Energy-aware scheduling: Kernel Summit Workshop Update. In Linaro Connect USA (LCU'14).

Lina Sawalha, Monte P. Tull, and Ronald D. Barnes. 2011. Thread scheduling for heterogeneous multicore processors using phase identification. SIGMETRICS Performance Evaluation Review 39, 3 (Dec. 2011), 125-127. DOI : http://dx.doi.org/10.1145/2160803.2160879

Filippo Sironi, Davide B. Bartolini, Simone Campanoni, Fabio Cancare, Henry Hoffmann, Donatella Sciuto, and Marco D. Santambrogio. 2012. Metronome: Operating system level performance management via self-adaptive computing. In Proceedings of the Design Automation Conference (DAC'12). IEEE, 856-865.

Vasileios Spiliopoulos, Stefanos Kaxiras, and Georgios Keramidas. 2011. Green governors: A framework for continuously adaptive DVFS. In Proceedings of the International Green Computing Conference and Workshops (IGCC'11). IEEE, 1-8.

Bo Su, Junli Gu, Li Shen, Wei Huang, Joseph L. Greathouse, and Zhiying Wang. 2014. PPEP: Online performance, power, and energy prediction framework and DVFS space exploration. In Proceedings of the IEEE / ACM International Symposium on Microarchitecture (MICRO-47'14). IEEE, 445-457.

Luís Taniça, Aleksandar Ilic, Pedro Tomás, and Leonel Sousa. 2014. SchedMon: A performance and energy monitoring tool for modern multi-cores. In Proceedings of the International Workshop on Multi/ManyCore Computing Systems (MuCoCoS/Euro-Par'14), Vol. 8806. Springer International Publishing, 230241.

Kenzo Van Craeynest, Aamer Jaleel, Lieven Eeckhout, Paolo Narvaez, and Joel Emer. 2012. Scheduling heterogeneous multi-cores through performance impact estimation (PIE). In Proceedings of the International Symposium on Computer Architecture (ISCA'12), Vol. 40. IEEE Computer Society, 213-224.

Augusto Vega, Alper Buyuktosunoglu, and Pradip Bose. 2013a. SMT-centric power-aware thread placement in chip multiprocessors. In Proceedings of the International Conference on Parallel Architectures and Compilation Techniques (PACT'13). IEEE, 167-176.

Augusto Vega, Alper Buyuktosunoglu, Heather Hanson, Pradip Bose, and Srinivasan Ramani. 2013b. Crank it up or dial it down: Coordinated multiprocessor frequency and folding control. In Proceedings of the Annual IEEE/ACM International Symposium on Microarchitecture (MICRO'13). ACM, New York, NY, 210-221.

Yuhao Zhu, Matthew Halpern, and Vijay Janapa Reddi. 2015. Event-based scheduling for energy-efficient QoS (eQoS) in mobile web applications. In Proceedings of the International Symposium on High Performance Computer Architecture (HPCA'15). IEEE, 137-149.

Received June 2015; revised October 2015; accepted October 2015 